\title{
ADAPTIVE IIR FILTERS FOR SINGLE INTERFERENCE SUPPRESSION IN A BPSK DS CDMA SYSTEM IN RAYLEIGH FADING CHANNEL
}

\author{
Aloys N. Mvuma \\ Telecommunications Engineering Department \\ University of Dar es Salaam \\ P.O. Box 35131 Dar es Salaam, TANZANIA \\ mvuma@udsm.ac.tz
}

\begin{abstract}
In this paper, effect of a single narrow-band interference (NBI) on bit error rate (BER) performance for a binary phase shift keying (BPSK) synchronous direct-sequence code-division multiple access (DS CDMA) communication system operating in a frequency nonselective Rayleigh fading channel is analyzed. Second-order adaptive infinite impulse response (IIR) notch filters with plain gradient algorithm (GA) for suppression of NBI in the DS CDMA system are proposed. A general closed-form BER expression for the DS CDMA system with NBI suppression second order adaptive IIR notch filters is derived based on the standard Gaussian approximation (SGA) method. BER expressions are then derived for the allpass filter-based adaptive IIR notch filter and adaptive IIR notch filter with constrained poles and zeros, the two structures that are commonly found in literature. It is observed that both adaptive IIR notch filter structures exhibit comparable BER performance. Extensive computer simulation results are presented to verify the accuracy and limitations of the analysis.
\end{abstract}

Keywords: Direct-sequence code-division multiple access, narrow-band interference, adaptive notch filters, bit error rate.

\section{INTRODUCTION}

Among the commonly used multiple access techniques in communication systems, directsequence code-division multiple-access (DS CDMA) is known to exhibit competitive advantages including the ability to combat multipath interference, increase system capacity, and improve quality of service (QoS) (Lee, 1991). Moreover, the combination of its low power spectral density (PSD) and its inherent immunity to narrowband interference (NBI) has been the basis for the proposed overlay systems that operate DS CDMA systems with existing narrowband users to improve the overall frequency spectrum utilization efficiency (Wang and Milstein, 1995). However, signal processing or suppression of NBI in DS CDMA systems caused by the existing narrow-band users is highly needed for proper performance of overlay systems.

Suppression of NBI for DS CDMA overlay systems using least-mean square (LMS)-based adaptive finite impulse response (FIR) line enhancers (FIRALEs) was proposed and its performance was extensively analyzed in (Poor and Rusch, 1994; Wang and Milstein, 1996; Laster and Reed, 1997). However, sharp notches are generally required for better performance, which necessitates the use of long and costly FIR-ALEs. On the other hand, it is well known that sharp notches can be easily achieved by adaptive infinite-impulse response (IIR) notch filters with considerably fewer coefficients and lower computational complexity compared to FIR-ALEs. It was for this reason that in recent years, suppression of NBI in DS CDMA systems using adaptive IIR notch filters was proposed and analyzed (Pei and Tseng, 1994; Mvuma et al., 2002; Jiang et al., 2002). Other NBI suppression methods found in literature include decision-directed adaptive filtering, adaptive analog-to-digital (A/D) conversion, nonlinear adaptive filtering, neural networks, etc. (Poor and Rusch, 1994; Chang and $\mathrm{Hu}, 1999)$.

In (Pei and Tseng, 1994; Mvuma et al., 2002; Jiang et al., 2002) an additive white Gaussian noise (AWGN) channel was assumed, which is not a realistic model for practical wireless applications. Moreover, in (Mvuma et al., 2002) only one structure, namely, an allpass filter-based secondorder adaptive IIR notch filter with gradient adaptation algorithm (GA) was considered. More research is therefore needed to establish performance capabilities and limitations of adaptive IIR notch filters in suppressing NBI in DS CDMA overlay systems.

The contribution of this paper is twofold. First, it studies an application of second-order adaptive IIR notch filters for suppression of NBI in a BPSK synchronous DS CDMA system operating in a frequency nonselective Rayleigh fading channel. This channel model is widely used in system design and performance analysis for DS-CDMA systems 
(Milstein et al., 1992; Monk et al., 1994). Secondly, a general bit error rate (BER) closed-form expression for the DS CDMA system with NBI suppression second-order adaptive IIR notch filter in terms of system parameters is derived. The derived expression can be used for analyzing the BER performance of any second-order adaptive IIR notch filter structure. For demonstration, closed form expressions are derived for the all pass filter-based adaptive IIR notch filter (Nishimura et el., 1989) and adaptive IIR notch filter with constrained poles and zeros (Xiao et al., 2001) and their performances are compared.

This paper is organized as follows. System and channel models that will be used throughout the paper are introduced in Section 2. Section 3 presents adaptive IIR notch filters and BER expressions. Simulation results and discussions are presented in Section 4, followed by concluding remarks in Section 5 .

\section{SYSTEM AND CHANNEL MODELS}

\section{Transmitter Model}

A binary phase shift keying (BPSK) DS CDMA system with $K$ active users is considered in this paper. A synchronous DS CDMA system is assumed, which is known to be an appropriate model for the forward link (base station to mobiles).
The transmitted signal for the $m$ th user, $s^{m}(t)$ is described by

$s^{m}(t)=\sqrt{2 P} b^{m}(t) c^{m}(t) \cos \left(2 \pi f_{c} t+\phi\right)$

where $P$ represents the transmitted signal power, assumed to be the same for all $K$ active users, $b^{m}(t)$ is the data signal, $c^{m}(t)$ is the spectrum spreading signal, $f_{c}$ is the carrier frequency and $\phi$ is the modulator carrier phase angle. The $m$ th user's data signal $b^{m}(t)$ is a rectangular waveform and is expressed as

$b^{m}(t)=\sum_{j=-\infty}^{\infty} b^{m}(j) P_{T_{b}}\left(t-j T_{b}\right)$

where $b^{m}(j)$ is the $j$ th data bit of the $m$ th user and is assumed to be random with equally probable values $\{+1,-1\}$,

$$
P_{T_{b}}(t)= \begin{cases}1, & 0 \leq t \leq T_{b} \\ 0, & \text { elsewhere }\end{cases}
$$

and $R_{b}=1 / T_{b}$ is the transmission bit rate. The spectrum spreading signal for the $m$ th user $c^{m}(t)$ can be expressed as

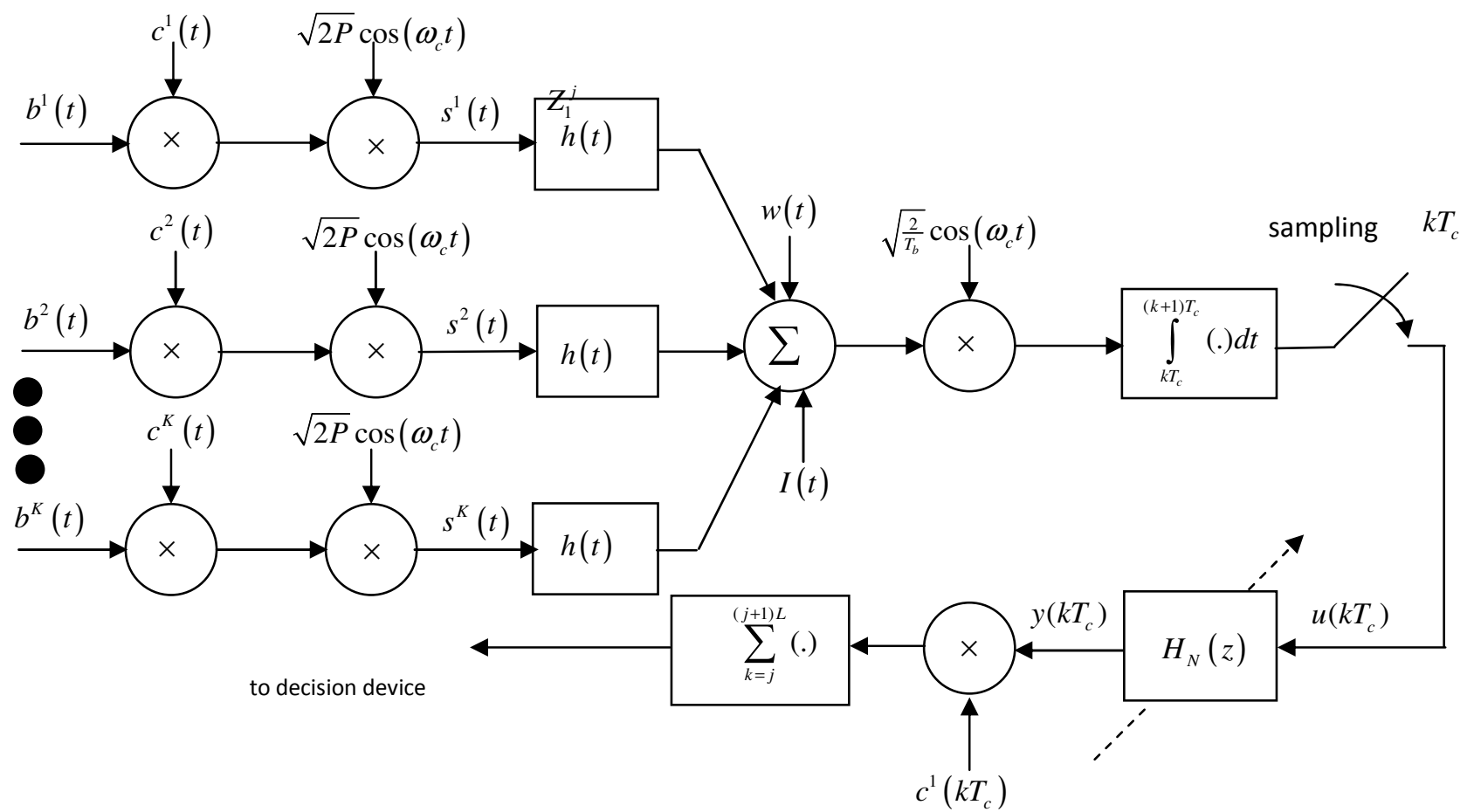

Figure 1. Synchronous DS CDMA system model in Rayleigh frequency nonselective channel 


$$
c^{m}(t)=\sum_{l=-\infty}^{\infty} c^{m}(l) P_{T_{c}}\left(t-l T_{c}\right)
$$

where

$$
P_{T_{c}}(t)= \begin{cases}1, & 0 \leq t \leq T_{c} \\ 0, & \text { elsewhere }\end{cases}
$$

and $c^{m}(l)$ is the $l$ th chip of the $m$ th user, which assumes values from $\{+1,-1\}$. In this paper, $\left\{c^{m}(l)\right\}_{m=1}^{K}$ are assumed to be maximal-length pseudonoise (PN) sequences of $\operatorname{period} N$. It is further assumed that there are $L$ chips in one data bit where $L<N$ for long codes and $L=N$ for short codes. $s^{m}(t)$ in (1) modifies to

$$
\begin{aligned}
& s^{m}(t)=\sqrt{2 P} \sum_{l=-\infty}^{\infty} b^{m}(\lfloor l / L\rfloor) c^{m}(l) P_{T_{c}}\left(t-l T_{c}\right) \\
& \times \cos \left(2 \pi f_{c} t+\phi\right)
\end{aligned}
$$

where $\lfloor x\rfloor$ denotes the largest integer less than $x$. It is noted from (6) that $j \square\lfloor l / L\rfloor=0$ for $l=0,1,2, \ldots, L-1 ; j=1$ for $l=L, L+1, L+2$, ..., $2 L-1$;

\section{Channel Model}

Signals for all active users, $\left\{s^{m}(t)\right\}_{m=1}^{K}$, are assumed to be transmitted over a frequency nonselective fading Rayleigh channel (Milstein et al., 1992; Monk et al., 1994). The channel impulse response is given by

$$
h(t)=\alpha e^{j \beta} \delta(t-\tau)
$$

The fading random variable $\alpha$ is Rayleighdistributed. It accounts for the fading channel attenuation of all signals and represents the envelope of a complex Gaussian process with unity variance in each quadrature component. The probability density function (pdf) of $\alpha$ is given by

$$
f_{A}(\alpha)=\alpha e^{-\alpha^{2} / 2}
$$

such that $E\left[\alpha^{2}\right]=1.0 . \beta$ in (7) is the phase introduced by the fading channel. It is assumed to be random and uniform over $(0,2 \pi) . \tau$ represents the time delay and accounts for the channel transmission delay. Fading channel parameters $\alpha, \beta$ and $\tau$ are assumed to be the same for all signals for the synchronous system.

The transmitted signal for the $m$ th user is further assumed to be corrupted with a zero-mean AWGN, $w(t)$ with two-sided power spectral density (PSD) $N_{0} / 2$ and NBI of unknown frequency, which is modeled by

$$
I(t)=\sqrt{2 J} \cos \left(\left\{\omega_{c}-\omega_{o}\right\} t+\theta\right)
$$

where $J$ is the NBI power, $\omega_{o}$ is the unknown offset from the carrier frequency and $\theta$ is the phase angle. The received signal $r(t)$ is thus given by

$$
\begin{aligned}
r(t)= & \sum_{m=1}^{K} s^{m}(t) * h(t)+w(t)+I(t) \\
= & \alpha \sqrt{2 P} \sum_{m=1}^{K} \sum_{l=-\infty}^{\infty} b^{m}(\lfloor l / L\rfloor) c^{m}(l) P_{T_{c}}\left(t-\tau-l T_{c}\right) \\
& \times \cos \left(2 \pi f_{c} t+\gamma\right)+w(t)+I(t)
\end{aligned}
$$

where $*$ denotes convolution and $\gamma=\beta+\phi-\omega_{c} \tau$ is assumed to be uniformly distributed over $(0,2 \pi)$.

\section{Receiver Model}

The fading is assumed to be slow such that conventional single-user matched filter coherent detection is used for the desired user signal. Here it is assumed that $m=1$ is the target user signal. The received signal $r(t)$ after conversion to baseband is passed through a chip matched filter and sampled at the end of every chip interval, i.e.

$$
\begin{gathered}
u\left(k T_{c}\right)=\sqrt{\frac{2}{T_{b}}} \int_{(k-1) T_{c}}^{k T_{c}} r(t) \cos \left(2 \pi f_{c} t+\gamma\right) d t \\
=u_{C D M A}\left(k T_{c}\right)+u_{I}\left(k T_{c}\right)+u_{w}\left(k T_{c}\right)
\end{gathered}
$$

$u_{C D M A}\left(k T_{c}\right)$ is the DS-CDMA signal comprised of the target user and the multiple access interference (MAI) caused by other $K-1$ active users and is expressed as

$$
u_{C D M A}\left(k T_{c}\right)=\alpha \frac{\sqrt{E_{b}}}{L} \sum_{l=-\infty}^{\infty} b^{m}(\lfloor l / L\rfloor) c^{m}\left(k T_{c}\right)
$$

where $E_{b}=P T_{b}$ is the average energy of the transmitted signal per bit for each active user. 
$u_{C D M A}\left(k T_{c}\right)$ is an uncorrelated random process with zero mean and variance $E\left[\alpha^{2}\right] K E_{b} / L^{2}$. $u_{I}\left(k T_{c}\right)$ is the NBI component and is expressed as

$$
u_{I}\left(k T_{c}\right)=\sqrt{J \frac{T_{c}}{L}} \operatorname{sinc}\left(f_{o} T_{c}\right) \cos \left(2 \pi f_{o} k T_{c}+\theta\right)
$$

Power of the NBI component in (13) is shown to depend on $f_{o}$ and will be zero whenever $f_{o} T_{c}=1,2, \ldots . \quad u_{w}\left(k T_{c}\right)$ is the noise component, which is expressed as

$$
u_{w}\left(k T_{c}\right)=\sqrt{\frac{2}{T_{b}}} \int_{k T_{c}}^{(1+k) T_{c}} w(t) \cos \left(2 \pi f_{c} t+\gamma\right) d t
$$

It can be shown that $u_{w}\left(k T_{c}\right)$ is an uncorrelated random process with zero-mean and variance $\sigma_{w}^{2}=N_{0} / 2 L$.

Referring to Fig. 1, if the adaptive notch filter $H_{N}(z)$ is not included, the receiver decision statistic, $Z^{1}(j)$ for the $j$ th data bit for the target user $(m=1)$ is given by

$$
\begin{aligned}
Z^{1}(j) & =\sum_{k=j}^{(j+1) L} u\left(k T_{c}\right) c^{1}(k) \\
= & \alpha \sqrt{E_{b}} b^{1}(j)+\alpha \frac{\sqrt{E_{b}}}{L} \sum_{k=j L}^{(j+1) L} \sum_{m=2}^{K} b^{m}(j) c^{m}(k) c^{1}(k) \\
& +\sum_{k=j L}^{(j+1) L} u_{I}\left(k T_{c}\right) c^{1}(k)+\sum_{k=j L}^{(j+1) L} u_{w}\left(k T_{c}\right) c^{1}(k)
\end{aligned}
$$

Using the central limit theorem, variance of the MAI in (15) averaged across the Rayleigh fading attenuation becomes

$$
\sigma_{M A I}^{2}=E\left[\alpha^{2}\right] E_{b} \frac{K-1}{L}
$$

Similarly, variances of the NBI and AWGN in (15) are given by

$$
\begin{gathered}
\sigma_{N B I}^{2}=\frac{J T_{c}}{2} \\
\sigma_{A W G N}^{2}=\frac{N_{0}}{2}
\end{gathered}
$$

Using the standard Gaussian approximation (SGA) (Morrow and Lehnert, 1989), the average BER given $\alpha$ for the target user is expressed as

$P_{e \mid \alpha}=Q\left(\sqrt{\frac{1}{\frac{E\left[\alpha^{2}\right]}{\alpha^{2}} \frac{K-1}{L}+\frac{N_{0}}{2 \alpha^{2} E_{b}}+\frac{J T_{c}}{2 \alpha^{2} E_{b}}}}\right)$

where $Q(z)=1 / \sqrt{2 \pi} \int_{z}^{\infty} e^{-y^{2} / 2} d y$. Averaging over $\alpha$, the average BER in Rayleigh fading approximates to

$P_{e}=\frac{1}{2}\left[1-\frac{1}{\sqrt{1+\frac{N_{0}}{E\left[\alpha^{2}\right] E_{b}}+\frac{K-1}{L}+\frac{J T_{c}}{E\left[\alpha^{2}\right] E_{b}}}}\right]$

By defining decision statistic signal-to-AWGN-ratio as $\quad \mathrm{SNR}=E\left[\alpha^{2}\right] E_{b} / N_{O}$ and signal-tointerference ratio (SIR) at the receiver input as $\mathrm{SIR}=E\left[\alpha^{2}\right] P / J$ (20) modifies to

$$
P_{e}=\frac{1}{2}\left[1-\frac{1}{\sqrt{1+\frac{1}{\mathrm{SNR}}+\frac{K-1}{L}+\frac{1}{L(\mathrm{SIR})}}}\right]
$$

It can be concluded from (21) that the effect of NBI on BER performance of DS CDMA system will be severely degraded when

$$
\mathrm{SIR}_{(\mathrm{dB})}<\mathrm{SNR}_{(\mathrm{dB})}-10 \log \left(\frac{K-1}{L}\right) .
$$

\section{ADAPTIVE IIR NOTCH FILTERS}

\section{Notch Filter Structures}

The NBI component, $u_{I}\left(k T_{c}\right)$ in (11) with unknown offset frequency $\omega_{o}$ can be tracked and suppressed using a second-order adaptive IIR notch filter with general transfer function $H_{N}(z)$. Suppression of NBI is intended to reduce its effect on the BER performance of the DS CDMA system. In this paper, allpass filter-based and constrained poles and zeros adaptive IIR notch filters are considered and their performances are compared. 


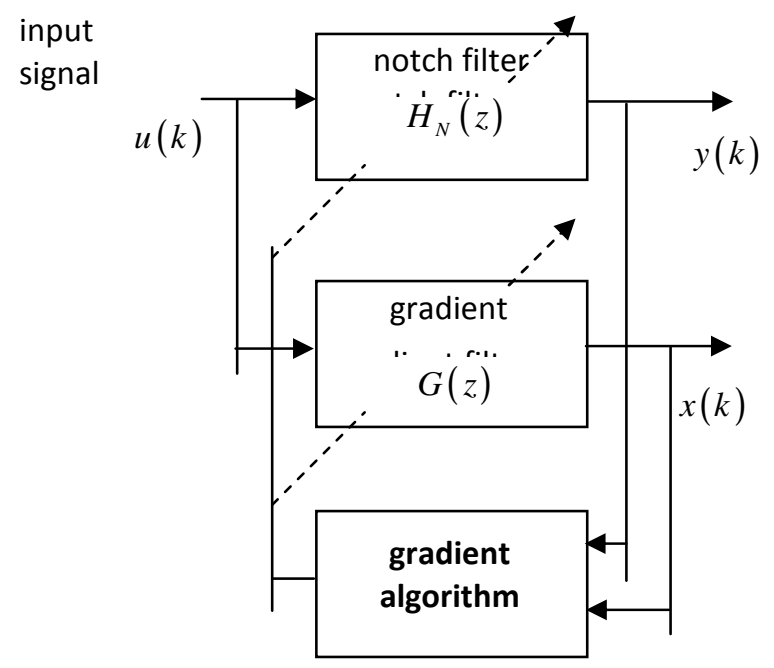

Figure 2. Second-order adaptive IIR notch filters block diagram.

\section{Allpass Filter-based Adaptive IIR Notch Filter}

Transfer function for the second order allpass filterbased adaptive IIR notch filter is given by (Nishimura et el., 1989)

$$
H_{N}(z)=\frac{1+\alpha_{0}}{2} \frac{1-2 \alpha_{1}(k) z^{-1}+z^{-2}}{1-\alpha_{1}(k)\left(1+\alpha_{0}\right) z^{-1}+\alpha_{0} z^{-2}}
$$

where $\alpha_{0}$ controls the notch bandwidth and pole radius $r$, i.e., $r=\sqrt{\alpha_{0}}$ and $\alpha_{1}$ determines the filter notch frequency $\omega_{n}$ according to $\alpha_{1}=\cos \left(\omega_{n}\right)$. Adaptive IIR Notch Filter with Constrained Poles and Zeros

Transfer function of the second order adaptive IIR notch filter with constrained poles and zeros is expressed as (Xiao et al., 2001)

$H_{N}(z)=\frac{1+\alpha_{1}(k) z^{-1}+z^{-2}}{1+\rho \alpha_{1}(k) z^{-1}+\rho^{2} z^{-2}}$

Similarly, $\rho$ controls the notch bandwidth and pole radius $r$ where $r=\rho$ and $\alpha_{1}=-2 \cos \left(\omega_{n}\right)$.

\section{Adaptation Algorithm}

The NBI in (11) is detected by adapting $\omega_{n}$ until it coincides with $\omega_{o}$ using the plain gradient algorithm (GA), and is formulated as (Nishimura et el., 1989; Xiao et al., 2001)

$\alpha_{1}(k+1)=\alpha_{1}(k)-\mu y(k) x(k)$
Here $\mu$ is the step size that controls the convergence speed, $y(k)$ is the output of the notch filter, $H_{N}(z)$ due to the input signal $u(k)$ in (11) ( $T_{c}$ is normalized to unity). $x(k)=\partial y(k) / \partial \alpha_{1}(k)$ is the gradient signal, which is the output of a gradient filter, $G(z)$.The transfer function for $G(z)$ depends on the notch filter structure where

$$
\begin{gathered}
G(z)=\left[H_{N}(z)-1\right] B(z) \\
B(z)=\frac{\left(1+\alpha_{0}\right) z^{-1}}{1-\alpha_{1}(k)\left(1+\alpha_{0}\right) z^{-1}+\alpha_{0} z^{-2}}
\end{gathered}
$$

and

$$
G(z) \approx z^{-1}-\rho z^{-1} H_{N}(z)
$$

for the allpass filter-based adaptive IIR notch filter and adaptive IIR notch filter with constrained poles and zeros, respectively. Fig. 2 shows the general configuration of the second-order adaptive IIR notch filter.

\section{Performance Analysis}

In Fig. 1, a decision variable $Z^{1}(j)$ for the 1 st user's $j$ th bit is shown to be obtained by correlating the notch filter output $y(k)$ with a locally generated PN sequence for user $1, c^{1}(k)$, which is assumed to be synchronized with that used at the transmitter. It should be noted here that $y(k)$ is obtained by convolving $u(k)$ with the 
impulse response $h_{N}(k)$ of the notch filter $H_{N}(z), \quad$ i.e., $y(k)=h_{N}(k) * u(k) . \quad$ It therefore follows that,

$$
\begin{aligned}
Z^{1}(j) & =\sum_{k=j}^{(j+1) L} y(k) c^{1}(k) \\
& =\alpha \sqrt{E_{b}} b^{1}(j) h(0)+\alpha \frac{\sqrt{E_{b}}}{L} \sum_{n=1}^{\infty} h_{N}(n) C_{L}(n) b^{1}\left(\left\lfloor\frac{k-n}{L}\right\rfloor\right) \\
& +\alpha \frac{\sqrt{E_{b}}}{L} \sum_{m=2}^{K} b^{m}\left(\left\lfloor\frac{k-l}{L}\right\rfloor\right) \sum_{n=0}^{\infty} h_{N}(n) \sum_{k=1}^{L} c^{1}(k) c^{m}(k-n) \\
& +\sum_{k=j}^{(j+1) L} c^{1}(k) \sum_{n=0}^{\infty} h_{N}(n) u_{I}(k-n) \\
& +\sum_{k=j}^{(j+1) L} c^{1}(k) \sum_{n=0}^{\infty} h_{N}(n) u_{w}(k-n)
\end{aligned}
$$

The first term on the right-hand side of (27) is the reference user's data component. The second term is the inter symbol interference (ISI) component caused by the notch filter. The third term is due to MAI whereas the fourth and fifth terms are due to the NBI and AWGN, respectively. $C_{L}(l)$ is the autocorrelation function of the PN sequence $c^{1}(k)$. Assuming $c^{m}(k)$ to be statistically independent random processes each with equally probable amplitudes $[+1,-1]$, variance of $Z^{1}(j)$ can be shown to be

$$
\begin{aligned}
& \operatorname{var}\left[Z^{1}(j)\right] \\
& =E\left[\alpha^{2}\right] E_{b} \frac{(K-1)}{L} \sum_{n=0}^{\infty} h_{N}^{2}(n)+\sigma_{I_{N}}^{2}+\frac{N_{0}}{2} \sum_{n=0}^{\infty} h_{N}^{2}(n) \\
& +\frac{E\left[\alpha^{2}\right]}{L} E_{b}\left(1+\frac{1}{N}\right)\left(1-\frac{L}{N}\right) \sum_{n=1}^{\infty} h_{N}^{2}(n)
\end{aligned}
$$

The first term on the right-hand side of (28) is the notch filter generated noise. This term reduces to zero when short codes are used, i.e., $L=N$. The third term $\sigma_{I_{H}}^{2}$ is the steady-state notch filter output power due to $u_{I}(k)$ which is normally very small and can be neglected for large $K$. The remaining part is the sum of terms due to AWGN and MAI.

Referring to (27) and (28) and assuming that the decision variable $Z^{1}(j)$ has a Gaussian distribution function, general expression for BER of the DS CDMA system reference user with NBI suppression second-order adaptive IIR notch filter becomes

$$
\begin{aligned}
P_{e \mid \alpha} & =Q\left(\sqrt{\frac{h_{N}(0)^{2} \alpha^{2} E_{b}}{\operatorname{var}\left[Z^{1}(j)\right]}}\right) \\
& =Q\left(\sqrt{\frac{h_{N}(0)^{2} \alpha^{2} E_{b}}{A E\left[\alpha^{2}\right] E_{b} \frac{\tilde{c}_{L}}{L}+B\left\{E\left[\alpha^{2}\right] \frac{K-1}{L}+\frac{N_{0}}{2}\right\}+\sigma_{I_{H}}^{2}}}\right)
\end{aligned}
$$

where

$$
\begin{aligned}
& A=\sum_{n=1}^{\infty} h_{N}^{2}(n), B=\sum_{n=0}^{\infty} h_{N}^{2}(n) \\
& \tilde{C}_{L}=\left(1+\frac{1}{N}\right)\left(1-\frac{L}{N}\right) .
\end{aligned}
$$

Here, Gaussian approximation is based on the use of central limit theorem. Averaging over $\alpha$, we approximate the average BER in Rayleigh fading as

$$
=\frac{1}{2}\left[1-\frac{1}{\sqrt{1+\frac{B}{h_{N}(0)^{2}}\left\{\frac{N_{0}}{E\left[\alpha^{2}\right] E_{b}}+\frac{K-1}{L}\right\}+\frac{A}{h_{N}(0)^{2}} \frac{\tilde{C}_{L}}{L}+\frac{L \sigma_{l_{H}^{2}}^{2}}{E\left[\alpha^{2}\right] E_{b}(0)^{2}}}}\right]
$$

It should be noted here that (30) shows BER as a function of $A, B$, and $h_{N}(0)^{2}$ for any arbitrary second-order adaptive IIR notch filter where

$$
\begin{aligned}
& h(0)=\frac{1}{2 \pi j} \mathfrak{f} H(z) z^{-1} d z \\
& \sum_{l=0}^{\infty} h^{2}(l)=\frac{1}{2 \pi j} \mathfrak{\int} H_{N}(z) H_{N}\left(z^{-1}\right) z^{-1} d z
\end{aligned}
$$

Referring to (22), it follows that for the allpass filter-based adaptive IIR notch filter: -

$$
\frac{A}{h_{N}(0)^{2}}=\frac{1-\alpha_{0}}{1+\alpha_{0}}, \frac{B}{h_{N}(0)^{2}}=\frac{2}{1+\alpha_{0}}, h_{N}(0)=\frac{1+\alpha_{0}}{2}
$$

Similar expressions for the adaptive IIR notch filter with constrained poles and zeros can be obtained by referring to (23) and are given by: - 


$$
\begin{aligned}
& \frac{A}{h_{N}(0)^{2}} \\
& =\frac{1}{\rho^{2}}\left\{1-\frac{1-\rho}{1+\rho} \frac{\left(1+\rho^{2}\right)(1+\rho)^{2}-8 \rho^{2} \cos ^{2} \omega_{o}}{\left(\rho^{4}-2 \rho^{2} \cos 2 \omega_{o}+1\right)}-\rho^{2}\right\} \\
& \frac{A}{h_{N}(0)^{2}} \\
& \quad=\frac{1}{\rho^{2}}\left\{1-\frac{1-\rho}{1+\rho} \frac{\left(1+\rho^{2}\right)(1+\rho)^{2}-8 \rho^{2} \cos ^{2} \omega_{o}}{\left(\rho^{4}-2 \rho^{2} \cos 2 \omega_{o}+1\right)}\right\} \\
& h_{N}(0)=1.0
\end{aligned}
$$

Comparison between (33) and (34) shows that $A$ approaches zero while $B$ and $h_{N}(0)$ approach unity as the notch filter pole radius increases for both adaptive IIR notch filter structures. Moreover, for the same pole radius, simulation and analytical results have shown both adaptive notch filters to exhibit similar BER performance.

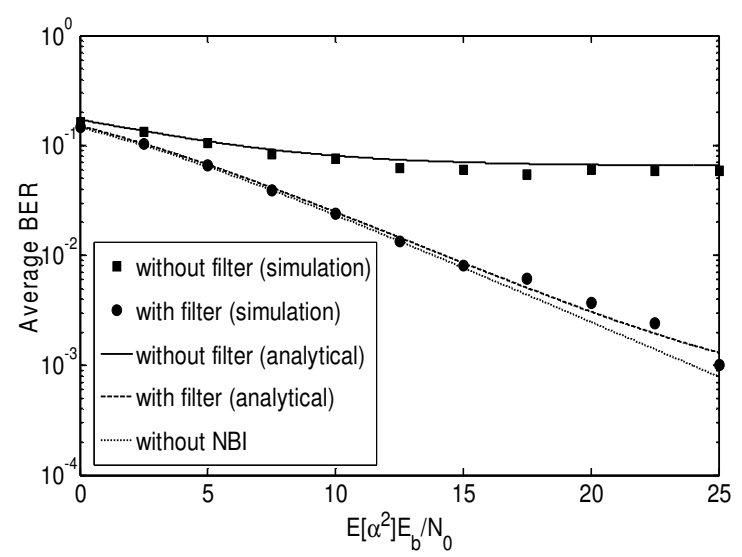

Figure 3. DS CDMA average BER versus $S N R$ for $\mathrm{SIR}=-10 \mathrm{~dB}$, pole radius $r=\sqrt{0.9}$, i.e., $\alpha_{0}=0.9$, $\rho=\sqrt{0.9}, K=1$ and $\omega_{o}=0.2 \pi$.

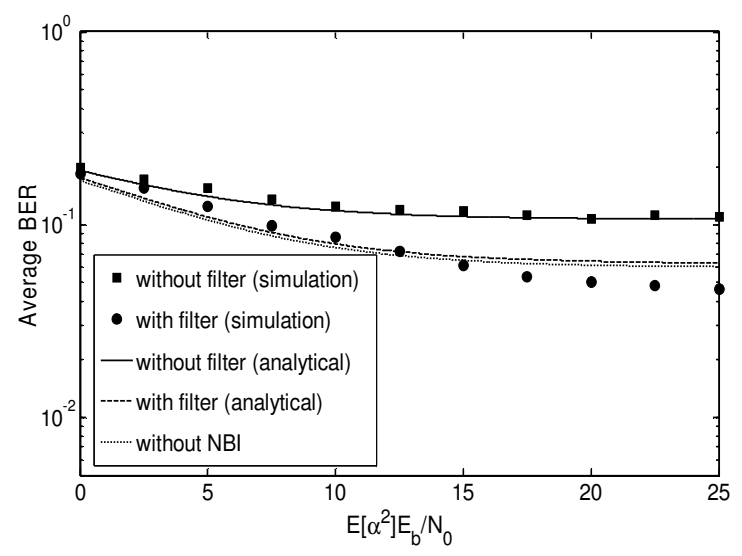

Figure 4. DS CDMA average BER versus SNR for $\mathrm{SIR}=-10 \mathrm{~dB}$, pole radius $r=\sqrt{0.9}$, i.e., $\alpha_{0}=0.9$, $\rho=\sqrt{0.9}, K=10$ and $\omega_{o}=0.2 \pi$.

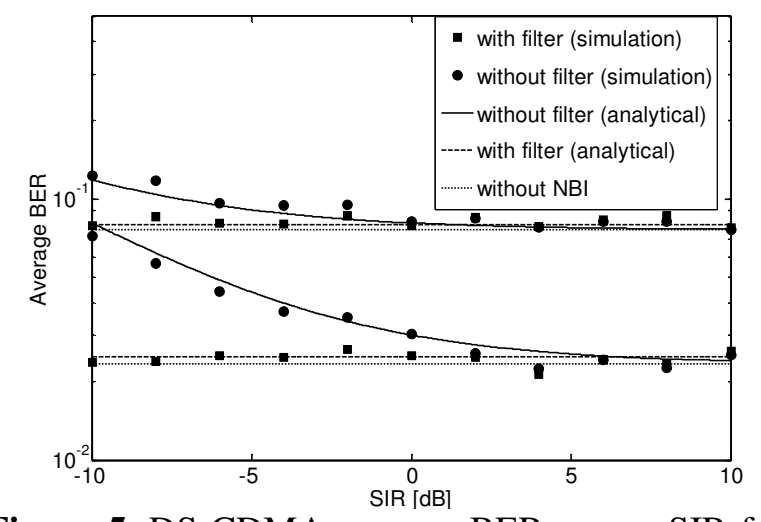

Figure 5. DS CDMA average BER versus SIR for $\mathrm{SNR}=10 \mathrm{~dB}$, pole radius $r=\sqrt{0.9}$, i.e., $\alpha_{0}=0.9$, $\rho=\sqrt{0.9}$ and $\omega_{o}=0.2 \pi$.

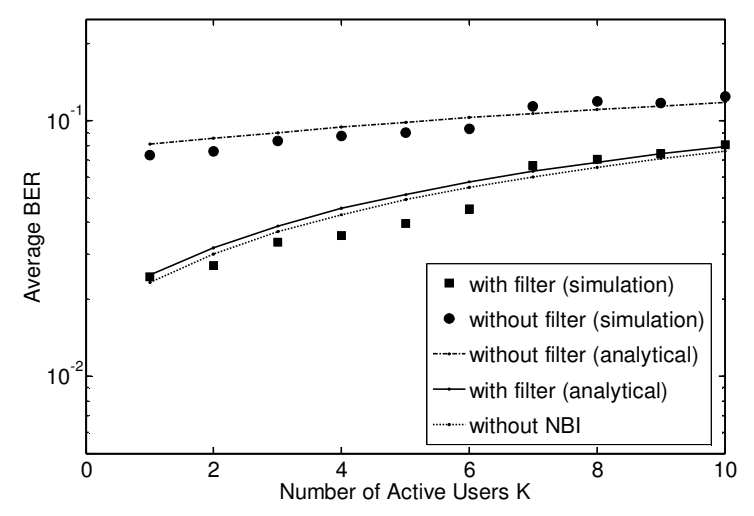

Figure 6. DS CDMA average BER versus number of active users $K$ for $\mathrm{SIR}=-10 \mathrm{~dB}, \mathrm{SNR}=10 \mathrm{~dB}$, pole radius $r=\sqrt{0.9}$, i.e., $\alpha_{0}=0.9, \rho=\sqrt{0.9}$, and $\omega_{o}=0.2 \pi$.

\section{SIMULATION RESULTS}

Simulation results are presented to support the theoretical findings. Step size $\mu$, pole radius $r$, and NBI offset frequency $\omega_{o}$ were fixed at 0.0005 , 0.9 , and $0.2 \pi$, respectively. PN m-sequences with period $N=255$ were used where $L$ was fixed to 31 and each user was assigned a unique sequence and $E_{b}$ was fixed at 1.0. Simulated BER were obtained by averaging over 10 independent computer runs for $10^{3}$ data bits.

Figures 3 and 4 show results for BER as a function of average SNR with SIR fixed at $-10 \mathrm{~dB}$ for $K=1$ and $K=10$, respectively. Close agreement 
between simulated and theoretical results is demonstrate by the figures. Moreover, significant BER improvement is shown to be gained by the use of NBI suppression adaptive IIR notch filters.

In Fig. 5, BER is shown plotted as a function of SIR with average SNR of $10 \mathrm{~dB}$ for $K=1$ and $K=10$. The figure shows the effect of NBI on the BER to be negligible for SIR $\geq 5 \mathrm{~dB}$ and SIR $\geq 10 \mathrm{~dB}$ for $K=10$ and $K=1$, respectively. Moreover, the NBI suppression adaptive IIR notch filters are shown to improve the BER for a wide range of SIR with very close agreement between theoretical and simulation results.

In Fig. 6, plots of average BER as a function of number of DS CDMA active users $K$ are shown for the average $S N R=10 \mathrm{~dB}$ and $\mathrm{SIR}=-10 \mathrm{~dB}$. Significant DS CDMA system capacity improvement is shown to be achievable by the use of NBI suppression adaptive IIR notch filters. Discrepancy between simulation and theoretical results in Fig. 5 for $K=2$ to $K=6$ is due to the inaccuracy of the SGA method that was used in deriving BER expressions.

\section{CONCLUSION}

Suppression of a single NBI in a synchronous BPSK DS CDMA communication system in a frequency non-selective Rayleigh fading channel using second-order adaptive IIR notch filters has been presented. General expression for BER has been derived and shown to be applicable for any adaptive IIR notch filter structure. Improvement in BER performance has been shown to be achievable by the use of adaptive IIR notch filters. Moreover, it has been found that for the same pole radius, the allpass filter-based adaptive IIR notch filter and adaptive IIR notch filter with constrained poles and zeros have equal BER performance. Adaptive IIR notch filters for suppression of multiple NBI in frequency non-selective and frequency selective Rician channels will be investigated in the future.

\section{REFERENCES}

Chang, P. R., and Hu, J. T., (1999). Narrow-band interference suppression in spread-spectrum CDMA communications using pipelined recurrent neural networks. IEEE Trans. Veh. Technol, vol. 48, no. 2, pp. 467 - 477.

Jiang, H. Y., Nishimura, S., and Hinamoto, T., (2002). Steady-state analysis of complex adaptive IIR notch filter and its application to QPSK communication systems. IEICE Trans. Fundamentals, vol. E85-A, no. 5, pp. $1088-1095$.

Laster, J. D., and Reed, J. H., (1997). Interference rejection in digital wireless communications. IEEE Signal Processing Magazine, vol. 14, no. 3 , pp. 37 - 62 .

Lee, W. C. Y., (1991). Overview of cellular CDMA. IEEE Trans. Veh. Technol., vol. 40, no. 3, pp. 291 - 302.

Milstein, L. B., Rappaport, T. S., and Barghouti, R., (1992). Performance evaluation for cellular CDMA. IEEE J. Select. Areas Commun., vol. 10 , pp. 680 - 689.

Monk, A. M., Davis, M., Milstein, L. B., and Helstrom, C. W., (1994). A noise whitening approach to multiple-access noise rejection Part 1: Theory and background. IEEE J. Select. Areas Commun., vol. 12, pp. 817 827.

Morrow, R. K., and Lehnert, J. S., (1989). Bit-to-bit error dependence in slotted DS/SSMA packet systems with random signature sequences. IEEE Trans. Commun., vol. 37, pp. 1052 - 1061.

Mvuma, A., Nishimura, S., and Hinamoto, T., (2002). Adaptive optimization of notch bandwidth of an IIR filter used to suppress narrow-band interference. Proc. IEEE International Symposium on Circuits and Systems (ISCAS), vol. V, pp. 341 - 344.

Nishimura, S., Kim, J. K., and Hirano, K., (1989). Mean-squared error analysis of an adaptive notch filter. Proc. IEEE International Symposium on Circuits and Systems (ISCAS), pp. 732 - 735.

Pei, S. C., and Tseng, C. C., (1994). Complex adaptive IIR notch filter algorithm and its applications. IEEE Trans. Circuits and Syst. II, vol. 41, no. 10, pp. 158 - 163.

Poor, H. V., and Rusch, L. A., (1994). Narrowband interference suppression in spread spectrum CDMA. IEEE Personal Commun., vol. 1, no. 3, pp. $14-27$.

Wang, J., and Milstein, L. B., (1995). CDMA overlay situations for microcellular mobile 
communications. IEEE Trans. Commun., vol. 43, pp. $603-614$.

Wang, J., and Milstein, L. B., (1996). Adaptive LMS filters for cellular CDMA overlay situations. IEEE Journal of Selected Areas in Commun., vol. 14, pp. 1548 - 1559.
Xiao, Y., Takeshita, Y., and Shida, K., (2001). Steady-state analysis of a plain gradient algorithm for a second-order adaptive IIR notch filter with constrained poles and zeros. IEEE Trans. Circuits Syst., vol. CAS-48, no. 7, pp. 733 - 740. 